\title{
ISM spectrum by cosmic dust?
}

\author{
Thomas V. Prevenslik \\ Discovery Bay, Hong Kong \\ email: thomas.prevenslik@gmail.com
}

\begin{abstract}
The interstellar medium (ISM) spectrum is usually explained by the response of dust particles (DPs) to the absorption of ultraviolet (UV) and visible (VIS) photons from nearby stars. With regard to the unidentified infrared (UIR) bands, the DPs are thought heated by UV and VIS photons to about $100 \mathrm{~K}$ thereby exciting the polycyclic aromatic hydrocarbons (PAHs). However,the UIR bands may be explained with the DPs at $2.7 \mathrm{~K}$. To wit, the UIR bands form by the direct excitation of PAHs by infrared (IR) radiation induced from the absorption of cosmic microwave background (CMB) radiation in DPs by quantum electrodynamics (QED).
\end{abstract}

Keywords. (ISM:) dust, ISM: lines and bands, infrared: ISM, cosmology: theory

\section{Introduction}

In the ISM, the UIR bands have been linked ( $\mathrm{Li} 2004$ ) to the heating of DPs by UV photons (Li \& Draine 2001). The UIR bands observed in UV-poor regions (Uchida, et al. 1998) was explained by (Li \& Draine 2002) with VIS photons alone assuming thermal emission from DPs heated to the same $100 \mathrm{~K}$ temperature assumed for UV photons in (Li \& Draine 2001). However,the assumed $100 \mathrm{~K}$ temperature of DPs may be fortuitous given not only are UV and VIS intensities not uniform in the ISM, but the only electromagnetic (EM) radiation ubiquitous to the ISM is the cosmic microwave background (CMB) at $2.7 \mathrm{~K}$.

\section{Theory and Analysis}

$\mathrm{CMB}$ radiation at $2.7 \mathrm{~K}$ may explain the ISM spectrum given a frequency up-conversion process in DPs. One such process is QED induced EM radiation (Prevenslik 2008). Finding analogy with creating photons of wavelength $\lambda$ by supplying EM energy to a quantum mechanical box having walls separated by $\lambda / 2$, the DPs having diameter $D$ produce QED photons of wavelength $2 D$. In the ISM, EM energy is supplied to DPs upon the absorption of CMB radiation (Mie 1908).

But Mie absorption efficiency is low in single interactions of CMB radiation with a DP (Bohren \& Huffman 1983). The ISM spectrum by CMB radiation therefore relies on multiple Mie absorptions and QED emissions in a continuous distribution of DPs of diameter $D$, each interaction successively increasing the frequency of QED emissions to produce the ISM spectrum at near unity Mie absorption efficiency (Prevenslik 2008).

Moreover, the Einstein specific heat of DPs depends on the vibration frequency of the absorbed CMB photon under total internal reflection (TIR) as it adjusts to the EM confinement imposed by the DP geometry. Since EM confinement occurs at TIR frequencies beyond the UV, the specific heats already very low at $2.7 \mathrm{~K}$ vanish during $\mathrm{CMB}$ photon absorption. Absent specific heat, the absorbed CMB radiation cannot be conserved by an increase in temperature (Prevenslik 2008). Instead, conservation of absorbed CMB radiation proceeds by producing a continuum of QED induced EM radiation within the DP, the IR content of which exciting the PAHs to produce the UIR bands. 
Beyond excluding Mie absorption efficiency, the classical Stefan-Boltzmann (SB) equation assumes the DP has specific heat $c_{\mathrm{p}}$ to conserve the absorbed blackbody (BB) radiation by an increase in temperature $T$. Since the Einstein specific heat $c_{\mathrm{p}}$ vanishes, the modified SB equation for DPs including Mie absorption efficiency $Q_{\mathrm{abs}}$ is,

$$
\sigma \pi D^{2} Q_{\mathrm{abs}} T_{\mathrm{BB}}^{4}-E_{\mathrm{p}} \frac{d N_{\mathrm{p}}}{d t}=M c_{\mathrm{p}} \frac{d T}{d t}=0
$$

where, $\sigma$ is the $\mathrm{SB}$ constant, $T_{\mathrm{BB}}$ is $2.7 \mathrm{~K}, Q_{\mathrm{abs}}$ is the Mie absorption efficiency taken to be unity in multiple interactions, $M$ is the DP mass, $d T / d t$ is rate of DP temperature change, $d N_{\mathrm{p}} / d t$ is the rate of QED induced photons produced having Planck energy $E_{\mathrm{p}}=$ $h c / 2 D$. Here, $h$ is Planck's constant, and $c$ is the speed of light.

\section{Discussion}

Historically, DPs having an entire heat capacity comparable to the Planck energy of a single UV or VIS photon were thought (Purcell 1976) to cause temperature fluctuations, and even more recently, the UIR bands have been explained (Li \& Draine 2002) by temperatures of about $100 \mathrm{~K}$ upon heating by absorbed VIS photons. In contrast, the QED induced IR continuum directly excites the PAHs to produce the UIR bands with the DPs remaining at $2.7 \mathrm{~K}$. Moreover, DP temperatures of $100 \mathrm{~K}$ from absorbed UV and VIS photons are unlikely because the DP specific heats already low at $2.7 \mathrm{~K}$ vanish as the CMB photons upon absorption vibrate at optical TIR frequencies.

\section{Conclusions}

The ISM spectrum is produced by QED induced EM radiation from the multiple interactions of a continuum of DP diameters. DPs always remain at $2.7 \mathrm{~K}$. Neither UV nor VIS photons from nearby stars are necessary to produce the UIR bands by heating DPs to temperatures of $100 \mathrm{~K}$. In fact, CMB radiation of DPs may produce in part the UV and VIS now thought to originate in nearby stars.

The ISM spectrum produced by QED induced EM radiation finds origin in the blueshift of CMB radiation in submicron DPs.

The converse of blueshift of CMB radiation in DPs is also true in the redshift of VIS light from distant galaxies claimed in Hubbles Law. The galaxy light observed on Earth and thought to be redshift by the Doppler effect in an expanding universe may be nothing more than numerous QED induced blue and redshift of galaxy light in DPs that is finally observed on Earth as redshift. Hence, the Hubble theory as experimental evidence for an expanding universe is therefore held in question by QED induced EM radiation in DPs.

\section{References}

Bohren, C. F. \& Huffman, D. R. 1983, Absorption and Scattering of Light by Small Particles, J. Wiley \& Sons, New York

Li, A. 2004, Astrophysics of Dust, ASP Conference Series, 309, 417

Li, A. \& Draine, B. T. 2001, ApJ, 554, 778

Li, A. \& Draine, B. T. 2002, ApJ, 572, 232

Mie, G. 1908, Ann. Phys., 330, 337

Prevenslik, T. V., 2008, www.nanoqed.net

Purcell, E. M. 1976, ApJ, 206, 685

Uchida, K. I., Selgren, K., \& Werner, M. W. 1998, ApJ (Letters), 493, L109 